\title{
Influencia del microhábitat sobre índices de calidad de agua en una quebrada de bosque montano, Junín, Perú
}

\author{
Influence of the microhabitat on water quality indexes in a montano forest \\ stream, Junín, Peru
}

\author{
Janet Isabel Sajamí Reymundo ${ }^{1}$, Ana Asunción Huamantinco Araujo ${ }^{2}$
}

Recibido: 08/01/2021 - Aprobado: 08/03/2021 - Publicado: 18/06/2021

\begin{abstract}
RESUMEN
Los macroinvertebrados acuáticos son ampliamente utilizados en los estudios ambientales principalmente en la bioindicación donde su adecuada utilización depende del conocimiento que se tenga de esta comunidad. El objetivo del presente estudio fue determinar la influencia del microhábitat sobre dos de los índices más utilizados para evaluar la calidad del agua BMWP/Col y ABI, para lo cual se evaluaron cuatro microhábitats (piedra, grava, musgo y hoja retenida en la corriente) colectándose 103 géneros distribuidos en 52 familias de macroinvertebrados acuáticos. De los cuatro microhábitats evaluados, hoja retenida en la corriente presentó la más alta riqueza y abundancia (90 géneros y 5069 individuos). Los resultados mostraron que los diferentes microhábitats tuvieron un efecto significativo sobre las métricas de bioindicación BMWP/Col y $A B I(p<0.05)$ asimismo las comparaciones múltiples post hoc evidenciaron diferencias significativas $(p<0.05)$ entre los microhábitats de piedra-hoja y musgo-hoja resaltando la importancia de un muestreo multihábitat. Se concluyó que tanto el índice BMWP/Col y ABI dependen de la heterogeneidad del hábitat y el uso de ambos se complementarían para determinar la calidad acuática en quebradas de un ecosistema montano.
\end{abstract}

Palabras claves: Bioindicación; bosque montano; calidad del agua; macroinvertebrados acuáticos; microhábitat.

\begin{abstract}
Aquatic macroinvertebrates are widely used in environmental studies, mainly in bioindication, where their use depends on the knowledge of this community. The objective of the present study was to determine the influence of the microhabitat on two of the most used indices to evaluate the water quality BMWP / Col and ABI, for which four microhabitats were evaluated (stone, gravel, moss and leaf retained in the stream) collecting a total of 103 genera distributed in 52 families of aquatic macroinvertebrates. Of the four microhabitats evaluated, leaf registered the greatest richness and abundance (90 genera and 5069 individuals). The results showed that the different microhabitats had a significant effect on the bioindication metrics $B M W P / C o l$ and $A B I(p<0.05)$, and multiple post hoc comparisons showed significant differences $(p<0.05)$ between the stone-leaf microhabitats. and moss-leaf highlighting the importance of multi-habitat sampling. It was concluded that both the BMWP/Col and ABI index depend on the heterogeneity of the habitat and the use of both would complement each other to determine the aquatic quality in streams of a montane ecosystem.
\end{abstract}

Keywords: Bioindication; montane forest; water quality; aquatic macroinvertebrates; microhabitat.

\footnotetext{
1 Universidad Nacional Mayor de San Marcos, Facultad de Ciencias Biológicas, Laboratorio de Invertebrados Acuáticos. Lima, Perú. E-mail: janetsajamireymundo@gmail.com - ORCID: https://orcid.org/0000-0001-8650-5592

2 Investigadora RENACYT - CONCYTEC (Grupo María Rostworowski - Nivel I). Universidad Nacional Mayor de San Marcos. Lima, Perú E-mail: ahuamantincoa1@unmsm.edu.pe - ORCID: https://orcid.org/0000-0001-6558-1326
} 


\section{INTRODUCCIÓN}

El uso de la comunidad de macroinvertebrados acuáticos como bioindicadores se remonta al siglo XIX (año 1848), según lo mencionado por Roldán-Pérez (2016) y aunque su uso se ha diseminado ampliamente esto no ha ido a la par con el conocimiento taxonómico y ecológico de los diferentes grupos de esta comunidad, siendo indispensable un conocimiento previo antes de su adecuada utilización. Asimismo, el uso de las métricas de bioindicación tomando en cuenta esta comunidad debe ser cuidadosamente revisada a fin de saber si es apropiado para el tipo de ambiente a evaluar, dado que cada métrica fue desarrollada para ecosistemas determinados y solo en algunos casos adaptados. Además, si bien la comunidad de macroinvertebrados acuáticos son ampliamente usados en la bioindicación, su rol principal es formar parte de la cadena trófica de los ecosistemas acuáticos (Thorp, J.H., 2018), desarrollar ciclos de vida, interactuar con su entorno biótico y abiótico, responder a cambios ambientales naturales y antropogénicos, etc.

La heterogeneidad del hábitat juega un rol importante, donde el arrastre desde las partes altas del río promueve la sedimentación y deposición cambiando las características fisiográficas del ambiente (Allan \& Castillo, 2007; Huaylinos Villalva et al., 2003) y que son determinantes en el establecimiento de esta comunidad acuática. La hoja retenida en la corriente representa el principal sustrato de un ecosistema acuáticos montano albergando la mayor riqueza y abundancia de esta comunidad, debido a que es una fuente extraordinaria de alimento y refugio para estos organismos (Wallace \& Webster, 1996; Ramírez et al., 1998; Huamantinco \& Nessimian, 1999; Baptista et al., 2001; Graça, 2001; M. A.S. Graça et al., 2001; Meza-S et al., 2012), además de ser ampliamente disponible en ecosistemas montanos. Si bien los hojarasca retenida en la corriente podría ser considerado el microhábitat principal se resalta la importancia de otros microhábitats tales como piedra, grava y musgo debido a las preferencias y especificidad de algunos grupos de invertebrados, siendo importante un reconocimiento previo del área de estudio para su evaluación. Respecto a lo mencionado, autores como Dávila-Recinos et al. (2019), encontraron un efecto significativo del microhábitat sobre la comunidad de macroinvertebrados acuáticos en los ríos La Labor y Tzununá, en la cuenca del lago Atitlán en Guatemala; así también Meza-S et al. (2012) encontraron efectos significativos del microhábitat sobre la comunidad de macroinvertebrados acuáticos resaltando la disponibilidad de la hojarasca en el río Chinchiná y las quebradas El Diamante y La Oliva, subcuenca alta del río Chinchiná en Colombia, señalando en ambos casos la importancia de la heterogeneidad del hábitat. Maestre \& Carranza (2018) también señalaron que una mayor diversidad de sustratos es apropiada para el desarrollo de esta comunidad.

En cuanto a la bioindicación, su uso se ha extendido ampliamente en los estudios ambientales como la minería, utilizando bacterias como bioindicadores para la remediación (Lovera, D., Zavaleta, A., Puente, L., Quiñones, J., Gagliufi, P., Coronado, R., Arias, V., Terrel, M., Revilla, A., Diego, J., Semino, 2008), hasta el uso de índices de integridad biótica que toma en cuenta a los peces. Los efectos adversos en un ambiente acuático involucran cambios en las estructuras de las comunidades acuáticas (Alba-Tercedor, 1996), desde las bacterias hasta los vertebrados como los peces; donde los macroinvertebrados acuáticos, dado su asociación con el sedimento, nos proveen información sobre dichos cambios y que de acuerdo a su sensibilidad nos permite obtener índices tales como el Biological Monitoring Working Party (BMWP) que fue inicialmente desarrollado por la Unión Europea y posteriormente adaptado para Colombia (BMWP/Col) basándose en puntuaciones asignadas a diferentes familias de acuerdo a su grado de sensibilidad y tolerancia (Roldán, 2003); y, el Índice Biótico Andino (ABI) propuesto por Acosta et al. (2009) para ríos altoandinos que se encuentren sobre los $2000 \mathrm{~m} \mathrm{s.} \mathrm{n.} \mathrm{m.} \mathrm{y}$ se basa en la sumatoria de puntuaciones asignadas a cada familia, similar al BMWP/Col.

El objetivo principal de este estudio fue evaluar la influencia del microhábitat (piedra, grava, musgo y hoja retenida en la corriente) sobre las métricas BMWP/Col según Roldán (2003) y el ABI según la propuesta de Acosta et al. (2009). Asimismo, se detalla la composición de la comunidad de macroinvertebrados acuáticos por microhábitats. Es importante mencionar que el conocimiento previo del hábitat acuático y de las comunidades que alberga permitirá tomar una adecuada decisión sobre el manejo de los ecosistemas.

\section{MÉTODOS}

\section{1. Área de estudio}

La evaluación tuvo lugar en la quebrada Pichita, coordenadas 8773232 N y 452228 E (World Geodetic System 1984) Zona $18 \mathrm{~L}$ a una altitud de 2295 m s. n. m. dentro de la Concesión para la Conservación del Bosque Puyu Sacha perteneciente al Fundo Vista Alegre de la Asociación Peruana para la Promoción del Desarrollo Sostenible (APRODES) en la Selva Central del Perú, distrito de San Ramón, provincia de Chanchamayo y departamento de Junín (Reynel, 2012).

El área de estudio se ubicó en la Zona de vida Bosque muy húmedo Montano bajo Tropical (bmh-MBT), según la clasificación ecológica desarrollada por Holdridge (2013); y a la ecorregión de Selva alta o Ceja de Selva según Brack Egg \& Mendiola (2000).

El Bosque Puyu Sacha se ubica al Sur del Santuario Nacional de Pampa Hermosa, presentando escasa intervención antrópica. Existen pocos registros climatológicos del lugar siendo la estación más cercana la estación de Huasahuasi según el Servicio Nacional de Meteorología e Hidrología del Perú (SENAMHI) reportándose una temperatura media de $19^{\circ} \mathrm{C}$ y una precipitación total anual promedio de $2100 \mathrm{~mm}$ (Reynel, 2012).

\subsection{Caracterización ambiental}

En cada campaña (bimestral) se midieron las variables fisicoquímicas considerándose las siguientes: $\mathrm{pH}$, oxígeno disuelto $(\mathrm{mg} / \mathrm{L})$, conductividad $(\mu \mathrm{S} / \mathrm{cm})$, salinidad (ppm), sólidos totales disueltos $(\mathrm{mg} / \mathrm{L})$, temperatura ambiental 
$\left({ }^{\circ} \mathrm{C}\right)$ y temperatura del agua $\left({ }^{\circ} \mathrm{C}\right)$. Para la medición del pH se usó un pH-metro PHD-T104, para la medición del oxígeno un Oxímetro HANNA HI 9146; y, para la medida de la conductividad, salinidad, sólidos totales disueltos, temperatura ambiental y temperatura del agua se usó un Conductímetro EXTECH. Además, se calculó la velocidad de la corriente $(\mathrm{m} / \mathrm{s})$ y el caudal $(\mathrm{L} / \mathrm{s})$.

\subsection{Recolección de muestras}

Se realizaron seis colectas bimestrales entre marzo del 2013 y enero del 2014. Los macroinvertebrados fueron extraídos mediante el uso de una red surber de $(30 \mathrm{~cm} \mathrm{x}$ $30 \mathrm{~cm}$ ) con luz de malla de $200 \mu \mathrm{m}$. Las muestras fueron fijadas en alcohol de $96^{\circ}$ y almacenadas en bolsas plásticas. Las muestras fueron obtenidas de cuatro microhábitats representativos a lo largo de la quebrada Pichita: piedra, grava, musgo y hoja retenida en la corriente, obteniéndose cinco réplicas por cada tipo de microhábitat y durante cada una de las 6 campañas de evaluación. La identificación fue realizada según las claves taxonómicas de Domínguez et al. (2006), Domínguez \& Fernández (2009), Epler (1995), Hamada et al. (2019), Manzo (2005), V. Manzo \& Archangelsky (2008), Merritt, R. W., K. W. Cummins \& Berg (2008), Ottoboni Segura et al. (2011), Passos et al. (2007), Ruiz-Moreno, J., Ospina-Torres, R., Gómez-Sierra, H., \& Riss (2000) y, Thorp, J.H. (2018).

\subsection{Análisis de datos}

Para el análisis estadístico se consideraron las cinco réplicas por cada microhábitat. Se obtuvo la riqueza a través del número de géneros y la abundancia a través del número de individuos. Se calcularon las métricas de bioindicación BMWP/Col y ABI según la propuesta de Acosta et al. (2009). Se hizo un análisis paramétrico de Análisis de varianza (ANOVA) de una vía, luego de probar el supuesto de normalidad según la prueba de KolmogorovSmirnov; además se realizaron comparaciones múltiples post hoc según el método de Games-Howell. Asimismo, se construyeron barras de error para graficar la variación promedio de los índices BMWP/Col y ABI en los cuatro microhábitats.

\section{RESULTADOS}

\subsection{Caracterización ambiental}

Respecto a la caracterización ambiental, los meses con más caudal y velocidad de la corriente fueron: marzo (2013), noviembre (2013) y enero (2014); mientras que los meses julio (2013) y setiembre (2013) fueron los más secos disminuyendo el caudal y la velocidad de la corriente. Las medidas fisicoquímicas hidrológicas no mostraron diferencias marcadas excepto el oxígeno disuelto que fue más bajo en el mes de julio (Tabla 1).

\subsection{Composición y riqueza}

La comunidad de macroinvertebrados acuáticos estuvo conformada por 103 géneros, agrupados en 52 familias y los siguientes órdenes taxonómicos: Amphipoda, Basommatophora, Coleoptera, Entomobryomorpha, Diptera, Ephemeroptera, Hemiptera, Lepidoptera, Odonata, Plecoptera, Trichoptera, Trombidiformes y un orden no determinado (clase Clitellata). De todos los órdenes registrados, Diptera presentó 46 géneros distribuidos en 15 familias. Seguidamente el orden Coleoptera presentó 25 géneros agrupados en 11 familias de las cuales la familia Elmidae registró la mayor riqueza con 10 géneros. El tercer orden más abundante fue Trichoptera con 15 géneros distribuidos en 15 familias (Tabla 2).

El microhábitat con mayor número de individuos de macroinvertebrados acuáticos fue hoja retenida en la corriente (5 069 individuos, 36\%) seguido de musgo (3 $497,25 \%$ ) y grava (3 214 individuos, $23 \%$ ) y en menor abundancia en piedra (2 365 individuos, 16\%) (Figura 1).

Respecto a los géneros que fueron los más abundantes según tipo de microhábitat se tuvo al género Gigantodax con un $14.4 \%$ en el microhábitat de piedra; a Farrodes (9.4\%), Anchytarsus (7.7\%) y Anacroneuria (7.5\%) en el microhábitat de grava; a Rheotanytarsus (19.4\%) en el microhábitat de musgo; y a Parametriocnemus (13.7\%) y Phylloicus (12.6\%) en el microhábitat de hoja (Figura 2).

\section{3. Índices de bioindicación}

La aplicación del análisis de varianza para conocer si hay diferencias en los índices según el tipo de microhábitat,

Tabla 1. Medidas fisicoquímicas e hidrológicas de la quebrada Pichita (marzo-2013 a enero-2014)

\begin{tabular}{|c|c|c|c|c|c|c|c|}
\hline \multirow{2}{*}{ Parámetros } & \multirow{2}{*}{ Unidades } & \multicolumn{5}{|c|}{2013} & \multirow{2}{*}{$\begin{array}{c}2014 \\
\text { Enero }\end{array}$} \\
\hline & & Marzo & Mayo & Julio & Setiembre & Noviembre & \\
\hline $\mathrm{pH}$ & unidades de $\mathrm{pH}$ & 7.07 & 7.98 & 7.88 & 7.8 & 7.64 & 7.45 \\
\hline Conductividad & $\mu S / \mathrm{cm}$ & 92.6 & 103.9 & 101 & 79.8 & 72.7 & 65.5 \\
\hline Salinidad & ppm & 45.5 & 50.7 & 50.6 & 40.2 & 36.2 & 32.3 \\
\hline Solidos Totales & $\mathrm{mg} / \mathrm{L}$ & 64.8 & 72.5 & 70.9 & 55.8 & 50.6 & 44.8 \\
\hline Oxígeno disuelto & $\mathrm{mg} / \mathrm{L}$ & 7.6 & 7.15 & 5.64 & 6.02 & 6.47 & 7.75 \\
\hline Temperatura ambiental & ${ }^{\circ} \mathrm{C}$ & 15.9 & 21.7 & 15.1 & 22.8 & 18.8 & 16.1 \\
\hline Temperatura del agua & ${ }^{\circ} \mathrm{C}$ & 13.7 & 13.9 & 13.6 & 13.7 & 14.3 & 14.7 \\
\hline Velocidad de la corriente & $\mathrm{m} / \mathrm{s}$ & 0.52 & 0.66 & 0.25 & 0.33 & 0.83 & 0.69 \\
\hline Caudal & $\mathrm{L} / \mathrm{s}$ & 127.3 & 52.83 & 4.92 & 33.56 & 218.6 & 224.6 \\
\hline
\end{tabular}


Tabla 2. Abundancia relativa (AR \%) de los macroinvertebrados acuáticos en la quebrada Pichita

\begin{tabular}{|c|c|c|c|c|c|c|c|c|c|}
\hline ORDEN & Género & Piedra & A.R. $\%$ & Grava & A.R. $\%$ & Musgo & A.R. $\%$ & Hoja & A.R. $\%$ \\
\hline \multicolumn{10}{|l|}{ Familia } \\
\hline \multicolumn{10}{|l|}{ AMPHIPODA } \\
\hline Hyalellidae & Hyalella & 0 & 0.00 & 2 & 0.06 & 0 & 0.00 & 0 & 0.00 \\
\hline \multicolumn{10}{|c|}{ BASOMMATOPHORA } \\
\hline Planorbidae & Planorbidae nd & 0 & 0.00 & 3 & 0.09 & 1 & 0.03 & 1 & 0.02 \\
\hline \multicolumn{10}{|l|}{ COLEOPTERA } \\
\hline Curculionidae & Curculionidae nd & 0 & 0.00 & 2 & 0.06 & 0 & 0.00 & 5 & 0.10 \\
\hline \multirow[t]{10}{*}{ Elmidae } & Austrolimnius & 7 & 0.30 & 78 & 2.43 & 6 & 0.17 & 3 & 0.06 \\
\hline & Cylloepus & 41 & 1.73 & 118 & 3.67 & 26 & 0.74 & 85 & 1.68 \\
\hline & Elmidae nd & 11 & 0.47 & 0 & 0.00 & 94 & 2.69 & 2 & 0.04 \\
\hline & Heterelmis & 23 & 0.97 & 41 & 1.28 & 188 & 5.38 & 223 & 4.40 \\
\hline & Hexanchorus & 0 & 0.00 & 0 & 0.00 & 2 & 0.06 & 0 & 0.00 \\
\hline & Huleechius & 6 & 0.25 & 74 & 2.30 & 3 & 0.09 & 12 & 0.24 \\
\hline & Macrelmis & 1 & 0.04 & 2 & 0.06 & 1 & 0.03 & 4 & 0.08 \\
\hline & Neoelmis & 38 & 1.61 & 146 & 4.54 & 9 & 0.26 & 36 & 0.71 \\
\hline & Onychelmis & 2 & 0.08 & 0 & 0.00 & 11 & 0.31 & 1 & 0.02 \\
\hline & Pseudodisersus & 1 & 0.04 & 12 & 0.37 & 3 & 0.09 & 21 & 0.41 \\
\hline Gyrinidae & Andogyrus & 0 & 0.00 & 4 & 0.12 & 0 & 0.00 & 2 & 0.04 \\
\hline Hydraenidae & Hydraena & 1 & 0.04 & 2 & 0.06 & 1 & 0.03 & 33 & 0.65 \\
\hline \multirow[t]{2}{*}{ Hydrophilidae } & Dactylosternum & 1 & 0.04 & 0 & 0.00 & 0 & 0.00 & 11 & 0.22 \\
\hline & Hydrophilidae nd & 4 & 0.17 & 0 & 0.00 & 5 & 0.14 & 14 & 0.28 \\
\hline Lampyridae & Lampyridae nd & 0 & 0.00 & 1 & 0.03 & 3 & 0.09 & 10 & 0.20 \\
\hline Lutrochidae & Lutrochus & 42 & 1.78 & 12 & 0.37 & 30 & 0.86 & 170 & 3.35 \\
\hline Psephenidae & Pheneps & 31 & 1.31 & 83 & 2.58 & 10 & 0.29 & 11 & 0.22 \\
\hline \multirow[t]{2}{*}{ Ptilodactylidae } & Ptilodactylidae nd & 13 & 0.55 & 4 & 0.12 & 28 & 0.80 & 71 & 1.40 \\
\hline & Anchytarsus & 19 & 0.80 & 248 & 7.72 & 1 & 0.03 & 10 & 0.20 \\
\hline Scirtidae & Cyphon & 4 & 0.17 & 4 & 0.12 & 33 & 0.94 & 178 & 3.51 \\
\hline \multirow[t]{4}{*}{ Staphilinidae } & Ambodina & 1 & 0.04 & 0 & 0.00 & 0 & 0.00 & 1 & 0.02 \\
\hline & Oxytelus & 0 & 0.00 & 0 & 0.00 & 0 & 0.00 & 5 & 0.10 \\
\hline & Paracyusa & 0 & 0.00 & 0 & 0.00 & 5 & 0.14 & 0 & 0.00 \\
\hline & Philonthus & 2 & 0.08 & 0 & 0.00 & 32 & 0.92 & 31 & 0.61 \\
\hline \multicolumn{10}{|c|}{ ENTOMOBRYOMORPHA } \\
\hline Entomobryidae & Entomobryidae nd & 25 & 1.06 & 12 & 0.37 & 17 & 0.49 & 99 & 1.95 \\
\hline \multicolumn{10}{|l|}{ DIPTERA } \\
\hline \multirow[t]{3}{*}{ Blephariceridae } & Blepharicerinae nd & 12 & 0.51 & 0 & 0.00 & 3 & 0.09 & 0 & 0.00 \\
\hline & Limonicola & 10 & 0.42 & 0 & 0.00 & 3 & 0.09 & 0 & 0.00 \\
\hline & Paltostoma & 6 & 0.25 & 0 & 0.00 & 5 & 0.14 & 0 & 0.00 \\
\hline Canaceae & Canacea & 0 & 0.00 & 0 & 0.00 & 0 & 0.00 & 11 & 0.22 \\
\hline \multirow[t]{5}{*}{ Ceratopogonidae } & Atrichopogon & 1 & 0.04 & 1 & 0.03 & 10 & 0.29 & 2 & 0.04 \\
\hline & Bezzia & 4 & 0.17 & 25 & 0.78 & 67 & 1.92 & 72 & 1.42 \\
\hline & Ceratopogonidae nd & 11 & 0.47 & 25 & 0.78 & 126 & 3.60 & 46 & 0.91 \\
\hline & Forcipomyia & 14 & 0.59 & 0 & 0.00 & 4 & 0.11 & 6 & 0.12 \\
\hline & Probezzia & 19 & 0.80 & 67 & 2.08 & 88 & 2.52 & 110 & 2.17 \\
\hline \multirow[t]{4}{*}{ Chironomidae } & Chironomidae nd & 8 & 0.34 & 17 & 0.53 & 18 & 0.51 & 23 & 0.45 \\
\hline & Corynoneura & 6 & 0.25 & 3 & 0.09 & 48 & 1.37 & 67 & 1.32 \\
\hline & Cricotopus & 48 & 2.03 & 100 & 3.11 & 47 & 1.34 & 131 & 2.58 \\
\hline & Dicrotendipes & 3 & 0.13 & 10 & 0.31 & 0 & 0.00 & 1 & 0.02 \\
\hline
\end{tabular}




\begin{tabular}{|c|c|c|c|c|c|c|c|c|c|}
\hline ORDEN & Género & Piedra & A.R. $\%$ & Grava & A.R. $\%$ & Musgo & A.R. $\%$ & Hoja & A.R. $\%$ \\
\hline & Larsia & 5 & 0.21 & 9 & 0.28 & 4 & 0.11 & 7 & 0.14 \\
\hline & Metriocnemus & 2 & 0.08 & 2 & 0.06 & 0 & 0.00 & 22 & 0.43 \\
\hline & Nanocladius & 1 & 0.04 & 2 & 0.06 & 0 & 0.00 & 17 & 0.34 \\
\hline & Onconeura & 11 & 0.47 & 6 & 0.19 & 19 & 0.54 & 38 & 0.75 \\
\hline & Parametriocnemus & 51 & 2.16 & 52 & 1.62 & 137 & 3.92 & 695 & 13.71 \\
\hline & Pentaneura & 14 & 0.59 & 15 & 0.47 & 18 & 0.51 & 39 & 0.77 \\
\hline & Polypedilum & 13 & 0.55 & 21 & 0.65 & 4 & 0.11 & 31 & 0.61 \\
\hline & Rheocricoptopus & 1 & 0.04 & 5 & 0.16 & 0 & 0.00 & 0 & 0.00 \\
\hline & Rheotanytarsus & 86 & 3.64 & 13 & 0.40 & 679 & 19.42 & 42 & 0.83 \\
\hline & Stenochironomus & 3 & 0.13 & 1 & 0.03 & 3 & 0.09 & 5 & 0.10 \\
\hline & Stictocladius & 0 & 0.00 & 8 & 0.25 & 0 & 0.00 & 4 & 0.08 \\
\hline & Tanytarsus & 22 & 0.93 & 139 & 4.32 & 127 & 3.63 & 52 & 1.03 \\
\hline Dixidae & Dixela & 9 & 0.38 & 3 & 0.09 & 1 & 0.03 & 45 & 0.89 \\
\hline Dolichopodidae & Dolichopodidae nd & 0 & 0.00 & 0 & 0.00 & 2 & 0.06 & 7 & 0.14 \\
\hline \multirow[t]{2}{*}{ Empididae } & Hemerodromia & 3 & 0.13 & 0 & 0.00 & 1 & 0.03 & 0 & 0.00 \\
\hline & Neoplasta & 31 & 1.31 & 30 & 0.93 & 99 & 2.83 & 166 & 3.27 \\
\hline Muscidae & Muscidae nd & 0 & 0.00 & 0 & 0.00 & 1 & 0.03 & 2 & 0.04 \\
\hline \multirow[t]{2}{*}{ Psychodidae } & Maruina & 32 & 1.35 & 3 & 0.09 & 14 & 0.40 & 4 & 0.08 \\
\hline & Psychoda & 0 & 0.00 & 0 & 0.00 & 9 & 0.26 & 77 & 1.52 \\
\hline \multirow[t]{2}{*}{ Simuliidae } & Gigantodax & 340 & 14.38 & 55 & 1.71 & 67 & 1.92 & 36 & 0.71 \\
\hline & Simulium & 135 & 5.71 & 82 & 2.55 & 123 & 3.52 & 125 & 2.47 \\
\hline Stratiomyidae & Myxosargus & 0 & 0.00 & 1 & 0.03 & 0 & 0.00 & 3 & 0.06 \\
\hline \multirow[t]{2}{*}{ Tabanidae } & Chrysops & 0 & 0.00 & 2 & 0.06 & 2 & 0.06 & 0 & 0.00 \\
\hline & Tabanus & 0 & 0.00 & 1 & 0.03 & 1 & 0.03 & 0 & 0.00 \\
\hline Tanyderidae & Tanyderidae nd & 1 & 0.04 & 0 & 0.00 & 0 & 0.00 & 19 & 0.37 \\
\hline Tipulidae & Tipula & 0 & 0.00 & 5 & 0.16 & 17 & 0.49 & 55 & 1.09 \\
\hline \multirow[t]{7}{*}{ Limoniidae } & Cryptolabis & 3 & 0.13 & 3 & 0.09 & 0 & 0.00 & 0 & 0.00 \\
\hline & Dactylolabis & 7 & 0.30 & 5 & 0.16 & 22 & 0.63 & 39 & 0.77 \\
\hline & Hexatoma & 1 & 0.04 & 28 & 0.87 & 2 & 0.06 & 8 & 0.16 \\
\hline & Limnophila & 2 & 0.08 & 199 & 6.19 & 5 & 0.14 & 24 & 0.47 \\
\hline & Limonia & 1 & 0.04 & 5 & 0.16 & 6 & 0.17 & 24 & 0.47 \\
\hline & Limoniinae & 0 & 0.00 & 2 & 0.06 & 3 & 0.09 & 2 & 0.04 \\
\hline & Molophilus & 0 & 0.00 & 0 & 0.00 & 23 & 0.66 & 10 & 0.20 \\
\hline \multicolumn{10}{|c|}{ EPHEMEROPTERA } \\
\hline \multirow[t]{2}{*}{ Baetidae } & Baetodes & 188 & 7.95 & 61 & 1.90 & 69 & 1.97 & 10 & 0.20 \\
\hline & Mayobaetis & 71 & 3.00 & 50 & 1.56 & 68 & 1.94 & 109 & 2.15 \\
\hline \multirow[t]{2}{*}{ Leptohyphidae } & Haplohyphes & 21 & 0.89 & 104 & 3.24 & 5 & 0.14 & 19 & 0.37 \\
\hline & Leptohyphes & 9 & 0.38 & 16 & 0.50 & 11 & 0.31 & 30 & 0.59 \\
\hline \multirow[t]{3}{*}{ Leptophlebiidae } & Atopophlebia & 0 & 0.00 & 1 & 0.03 & 0 & 0.00 & 0 & 0.00 \\
\hline & Farrodes & 144 & 6.09 & 303 & 9.43 & 70 & 2.00 & 293 & 5.78 \\
\hline & Thraulodes & 15 & 0.63 & 48 & 1.49 & 1 & 0.03 & 2 & 0.04 \\
\hline \multicolumn{10}{|l|}{ HEMIPTERA } \\
\hline Hydrometridae & Bacillometra & 0 & 0.00 & 0 & 0.00 & 1 & 0.03 & 2 & 0.04 \\
\hline \multicolumn{10}{|l|}{ LEPIDOPTERA } \\
\hline Crambidae & Petrophila & 2 & 0.08 & 8 & 0.25 & 7 & 0.20 & 1 & 0.02 \\
\hline \multicolumn{10}{|l|}{ ND } \\
\hline ND & Clitellata nd & 4 & 0.17 & 12 & 0.37 & 71 & 2.03 & 8 & 0.16 \\
\hline ODONATA & & & & & & & & & \\
\hline
\end{tabular}




\begin{tabular}{|c|c|c|c|c|c|c|c|c|c|}
\hline ORDEN & Género & Piedra & A.R. $\%$ & Grava & A.R. $\%$ & Musgo & A.R. $\%$ & Hoja & A.R. $\%$ \\
\hline \multirow[t]{2}{*}{ Aeschnidae } & Allopetalia & 3 & 0.13 & 4 & 0.12 & 2 & 0.06 & 15 & 0.30 \\
\hline & Rhionaeschna & 0 & 0.00 & 9 & 0.28 & 2 & 0.06 & 3 & 0.06 \\
\hline Corduliidae & Neocordulia & 1 & 0.04 & 0 & 0.00 & 1 & 0.03 & 13 & 0.26 \\
\hline Polythoridae & Polythore & 18 & 0.76 & 22 & 0.68 & 15 & 0.43 & 32 & 0.63 \\
\hline \multicolumn{10}{|l|}{ PLECOPTERA } \\
\hline Perlidae & Anacroneuria & 97 & 4.10 & 242 & 7.53 & 60 & 1.72 & 278 & 5.48 \\
\hline \multicolumn{10}{|l|}{ TRICHOPTERA } \\
\hline Anomalopsychidae & Contulma & 0 & 0.00 & 10 & 0.31 & 9 & 0.26 & 12 & 0.24 \\
\hline Atriplectididae & Neoatriplectides & 0 & 0.00 & 1 & 0.03 & 0 & 0.00 & 0 & 0.00 \\
\hline Calamoceratidae & Phylloicus & 39 & 1.65 & 83 & 2.58 & 24 & 0.69 & 637 & 12.57 \\
\hline Glossosomatidae & Mortoniella & 177 & 7.48 & 28 & 0.87 & 12 & 0.34 & 4 & 0.08 \\
\hline Helicopsychidae & Helicopsyche & 124 & 5.24 & 109 & 3.39 & 32 & 0.92 & 22 & 0.43 \\
\hline Hydrobiosidae & Atopsyche & 9 & 0.38 & 12 & 0.37 & 20 & 0.57 & 4 & 0.08 \\
\hline \multirow[t]{2}{*}{ Hydropsychidae } & Leptonema & 143 & 6.05 & 195 & 6.07 & 45 & 1.29 & 154 & 3.04 \\
\hline & Smicridea & 27 & 1.14 & 6 & 0.19 & 157 & 4.49 & 17 & 0.34 \\
\hline \multirow[t]{2}{*}{ Hydroptilidae } & Metrichia & 55 & 2.33 & 2 & 0.06 & 420 & 12.01 & 5 & 0.10 \\
\hline & Neotrichia & 0 & 0.00 & 1 & 0.03 & 9 & 0.26 & 0 & 0.00 \\
\hline Leptoceridae & Nectopsyche & 2 & 0.08 & 8 & 0.25 & 49 & 1.40 & 243 & 4.79 \\
\hline Odontoceridae & Marilia & 11 & 0.47 & 62 & 1.93 & 3 & 0.09 & 17 & 0.34 \\
\hline Philopotamidae & Chimarra & 4 & 0.17 & 4 & 0.12 & 6 & 0.17 & 8 & 0.16 \\
\hline Polycentropodidae & Polycentropus & 1 & 0.04 & 2 & 0.06 & 0 & 0.00 & 1 & 0.02 \\
\hline Xiphocentronidae & Xiphocentron & 2 & 0.08 & 0 & 0.00 & 2 & 0.06 & 0 & 0.00 \\
\hline \multicolumn{10}{|l|}{ TROMBIDIFORMES } \\
\hline Limnesiidae & Limnesiidae nd & 9 & 0.38 & 18 & 0.56 & 9 & 0.26 & 24 & 0.47 \\
\hline Número de individuos & & 2365 & 100 & 3214 & 100 & 3497 & 100 & 5069 & 100 \\
\hline Número de géneros & & 80 & & 82 & & 87 & & 90 & \\
\hline
\end{tabular}

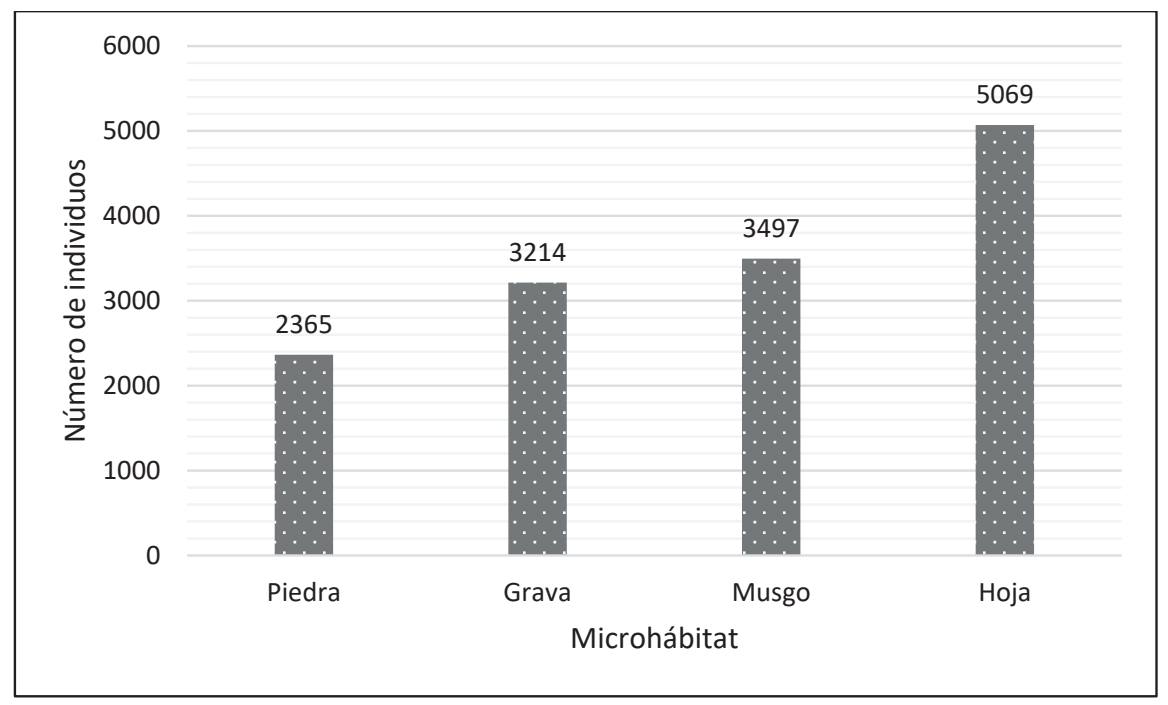

Figura 1. Abundancia relativa de la comunidad de macroinvertebrados acuáticos según microhábitats (piedra, grava, musgo y hoja). 
Los resultados del Anova de una vía se muestran en la Tabla 3, donde se encontró diferencias estadísticamente significativas con el índice $\mathrm{BMWP} / \mathrm{Col}(F=6.365 ; \mathrm{p}<$ $0.05)$ y el índice ABI $(F=6,512 ; \mathrm{p}<0.05)$. Asimismo, las comparaciones múltiples post hoc también evidenciaron diferencias estadísticamente significativas $(\mathrm{p}<0.05)$ entre los microhábitats de piedra-hoja y musgo-hoja tanto para el índice BMWP/Col y el índice ABI (Tabla 4). Los gráficos de barras de error (Figuras 3 y 4) muestran estas diferencias donde resalta el microhábitat de hoja retenida en la corriente con el más alto índice promedio respecto a los demás microhábitats. En cuanto a la calidad del agua en la quebrada Pichita esta varió entre "dudosa", "aceptable" y "buena" según el índice BMWP/Col; y, entre "moderada", "buena" y "muy buena" según el índice ABI; así mismo se registró los índices promedios más bajos en el mes de marzo (Tabla 5).

\section{DISCUSIÓN}

Los resultados hidrológicos en la quebrada Pichita evidenciaron una reducción del caudal y velocidad de la corriente entre julio y setiembre del año 2013. La temporalidad parece ser un patrón marcado en Perú con lluvias intensas durante el verano y con una tendencia a disminuir hacia los meses de mayo a noviembre. Resultados obtenidos en el río Cañete por Acosta Riveros (2009), en el río Utcubamba por

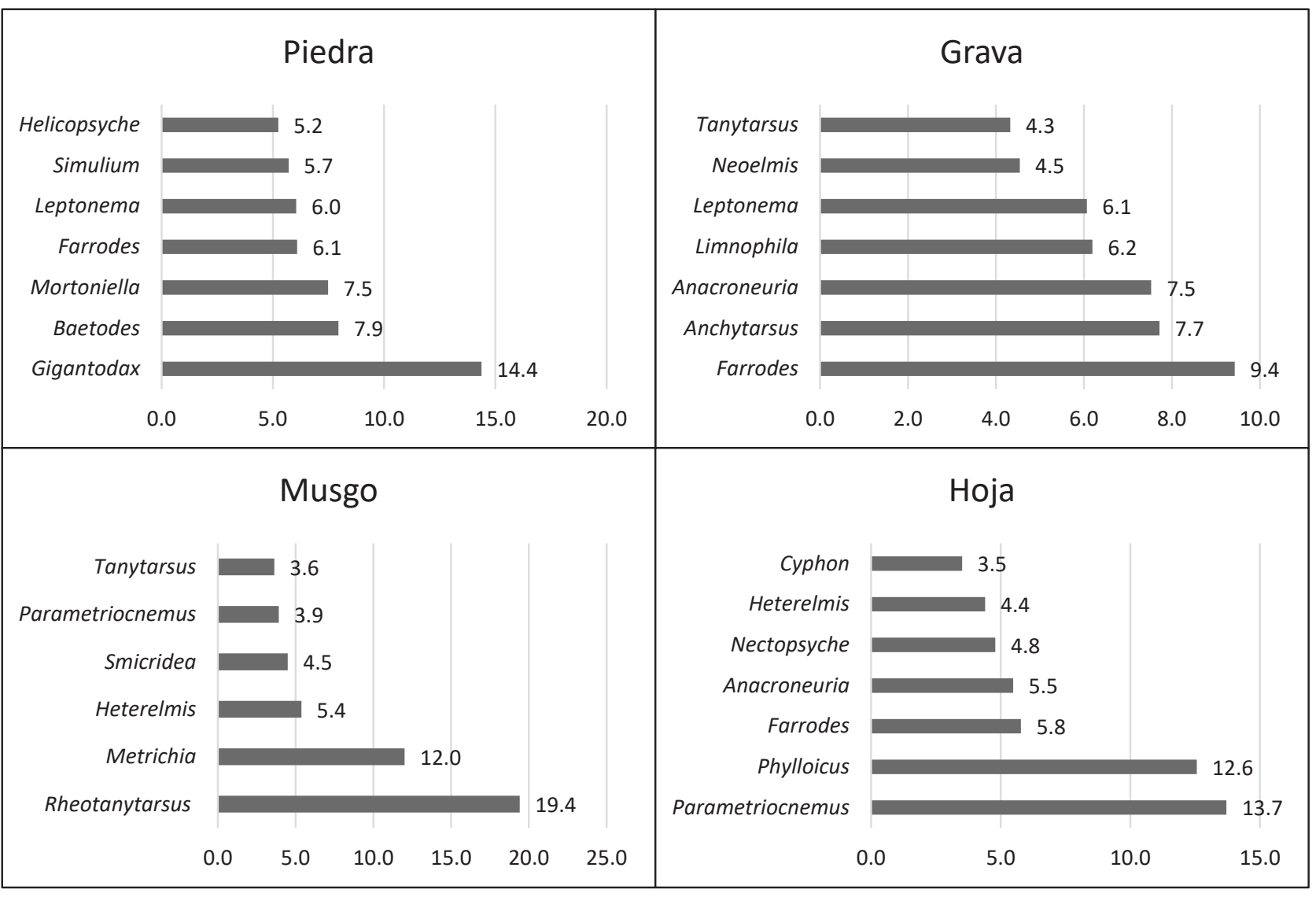

Figura 2. Abundancia relativa (A.R. \%) de los géneros de macroinvertebrados acuáticos más abundantes según microhábitats en la quebrada Pichita.

Tabla 3. Resultado del Anova de una vía (F, valor F de Fisher; gl, grados de libertad)

\begin{tabular}{|c|c|c|c|c|c|}
\hline \multirow{2}{*}{ Variable dependiente } & \multirow{2}{*}{ Microhábitats } & \multicolumn{4}{|c|}{ Estadísticos } \\
\hline & & Suma de cuadrados & gl & $F$ & $p$ \\
\hline \multirow{3}{*}{ BMWP/Col } & Entre grupos & 26901 & 3 & 6.365 & .001 \\
\hline & Dentro de grupos & 159184 & 113 & & \\
\hline & Total & 186084 & 116 & & \\
\hline \multirow{3}{*}{$A B I$} & Entre grupos & 20236 & 3 & 6.512 & .000 \\
\hline & Dentro de grupos & 117050 & 113 & & \\
\hline & Total & 137286 & 116 & & \\
\hline
\end{tabular}


ReV. INST. INVESTIG. FAC. MINAS METAL. CIENC. GEOGR. 24(47) 2021

Tabla 4. Comparaciones múltiples post hoc según el método de Games-Howell $(p<0.005)$

\begin{tabular}{|c|c|c|c|c|c|}
\hline $\begin{array}{l}\text { Variable } \\
\text { dependiente }\end{array}$ & & & $\begin{array}{l}\text { Diferencia de } \\
\text { medias }(\mathrm{I}-\mathrm{J})\end{array}$ & Error estándar & $p$ \\
\hline \multirow{12}{*}{ BMWP/Col } & \multirow{3}{*}{ Piedra } & Grava & -16.200 & 10.948 & .456 \\
\hline & & Musgo & -17.011 & 9.660 & .303 \\
\hline & & Hoja & $-41,900^{*}$ & 9.340 & .000 \\
\hline & \multirow{3}{*}{ Grava } & Piedra & 16.200 & 10.948 & .456 \\
\hline & & Musgo & -.811 & 10.156 & 1.000 \\
\hline & & Hoja & -25.700 & 9.851 & .056 \\
\hline & \multirow{3}{*}{ Musgo } & Piedra & 17.011 & 9.660 & .303 \\
\hline & & Grava & .811 & 10.156 & 1.000 \\
\hline & & Hoja & $-24,889^{*}$ & 8.397 & .023 \\
\hline & \multirow{3}{*}{ Hoja } & Piedra & $41,900^{*}$ & 9.340 & .000 \\
\hline & & Grava & 25.700 & 9.851 & .056 \\
\hline & & Musgo & $24,889^{*}$ & 8.397 & .023 \\
\hline \multirow{12}{*}{$A B I$} & \multirow{3}{*}{ Piedra } & Grava & -16.500 & 9.521 & .316 \\
\hline & & Musgo & -16.319 & 8.260 & .210 \\
\hline & & Hoja & $-36,633^{*}$ & 8.074 & .000 \\
\hline & \multirow{3}{*}{ Grava } & Piedra & 16.500 & 9.521 & .316 \\
\hline & & Musgo & .181 & 8.623 & 1.000 \\
\hline & & Hoja & -20.133 & 8.446 & .093 \\
\hline & \multirow{3}{*}{ Musgo } & Piedra & 16.319 & 8.260 & .210 \\
\hline & & Grava & -.181 & 8.623 & 1.000 \\
\hline & & Hoja & $-20,315^{*}$ & 6.993 & .026 \\
\hline & \multirow{3}{*}{ Hoja } & Piedra & $36,633^{*}$ & 8.074 & .000 \\
\hline & & Grava & 20.133 & 8.446 & .093 \\
\hline & & Musgo & $20,315^{*}$ & 6.993 & .026 \\
\hline
\end{tabular}

${ }^{*}$. La diferencia de medias es significativa en el nivel 0.05 .

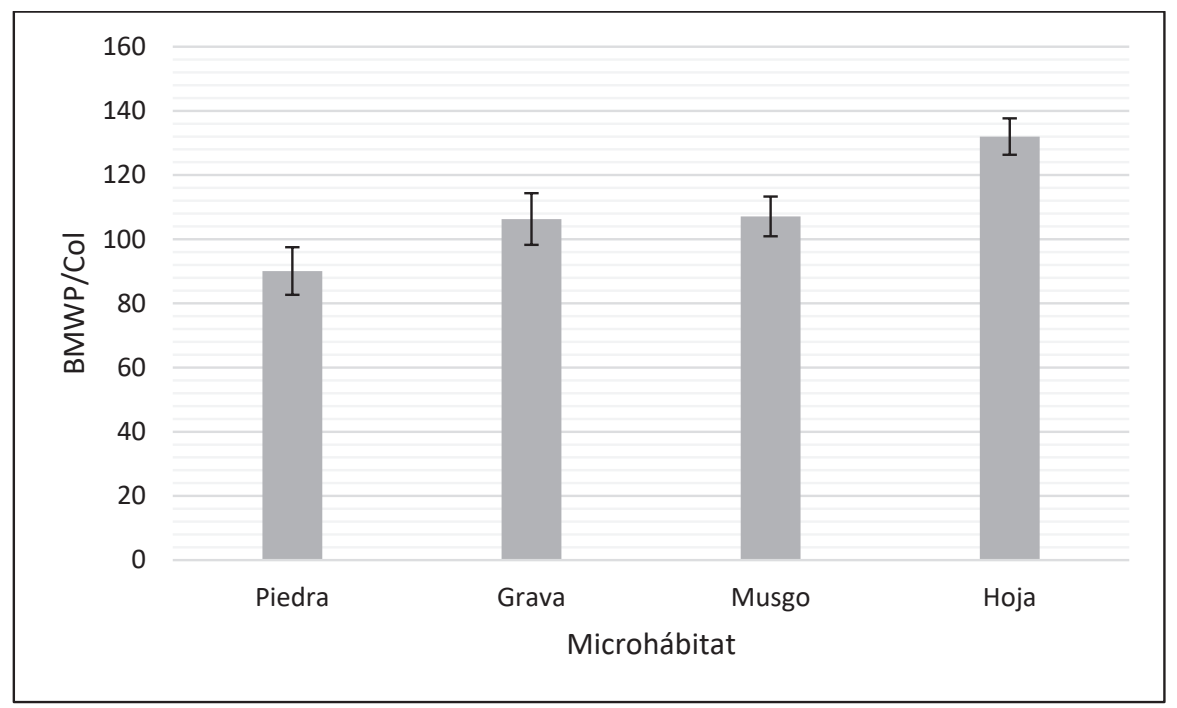

Figura 3. Barras de error típico del índice BMWP/Col según microhábitats de la quebrada Pichita. 


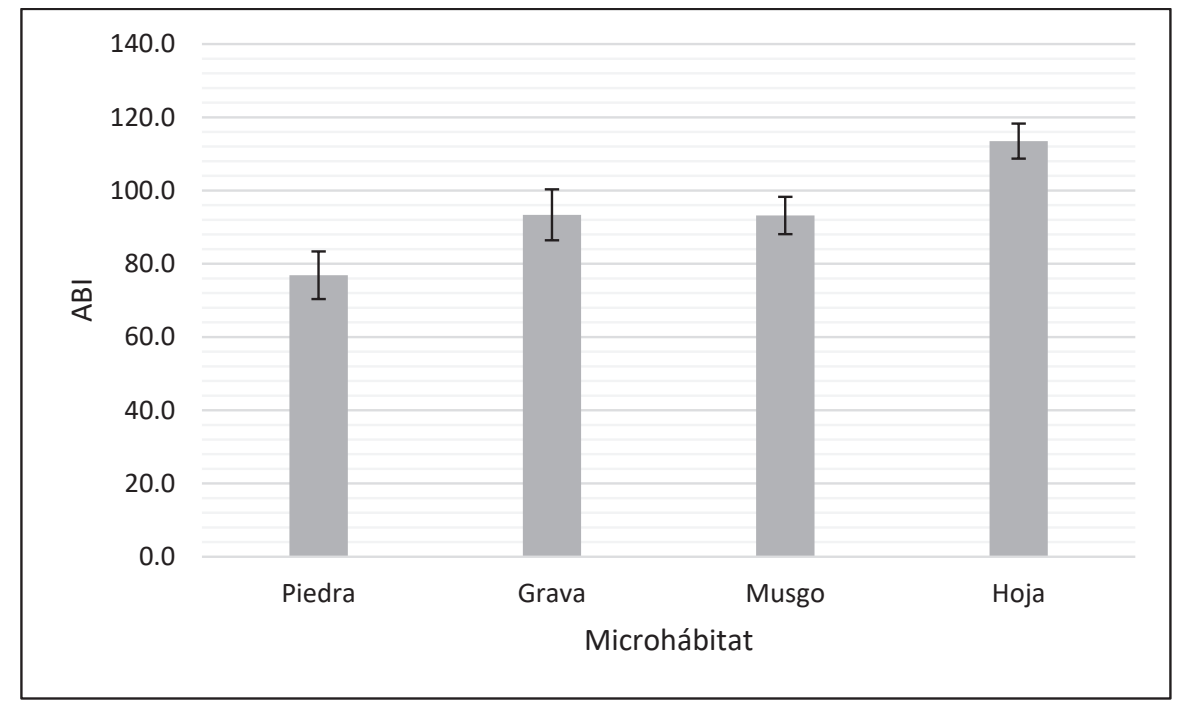

Figura 4. Barras de error típico del índice $A B I$ según microhábitats de la quebrada Pichita.

Tabla 5. Valores promedio y calidad del agua de los índices BMWP/Col y ABI según microhábitats y meses evaluados en la quebrada Pichita

\begin{tabular}{|c|c|c|c|c|c|c|c|c|}
\hline \multirow[b]{2}{*}{ Mes } & \multicolumn{2}{|c|}{ Piedra } & \multicolumn{2}{|c|}{ Grava } & \multicolumn{2}{|c|}{ Musgo } & \multicolumn{2}{|c|}{ Hoja } \\
\hline & $\begin{array}{l}\text { Valor } \\
\text { promedio }\end{array}$ & $\begin{array}{l}\text { Calidad del } \\
\text { agua }\end{array}$ & $\begin{array}{c}\text { Valor } \\
\text { promedio }\end{array}$ & $\begin{array}{l}\text { Calidad del } \\
\text { agua }\end{array}$ & $\begin{array}{c}\text { Valor } \\
\text { promedio }\end{array}$ & $\begin{array}{l}\text { Calidad del } \\
\text { agua }\end{array}$ & $\begin{array}{l}\text { Valor } \\
\text { promedio }\end{array}$ & $\begin{array}{c}\text { Calidad del } \\
\text { agua }\end{array}$ \\
\hline \multicolumn{9}{|c|}{ BMWP/Col } \\
\hline Marzo & 36.2 & Dudosa & 41.6 & Dudosa & 112.4 & Buena & 142.6 & Buena \\
\hline Mayo & 63.4 & Aceptable & 91.2 & Aceptable & 113.6 & Buena & 134.6 & Buena \\
\hline Julio & 155.4 & Buena & 124.2 & Buena & 107.5 & Buena & 129.8 & Buena \\
\hline Setiembre & 115.2 & Buena & 166.2 & Buena & 154.2 & Buena & 162.2 & Buena \\
\hline Noviembre & 88.6 & Aceptable & 124.6 & Buena & 66 & Aceptable & 99.8 & Aceptable \\
\hline Enero & 81.8 & Aceptable & 90 & Aceptable & 89.2 & Aceptable & 123 & Buena \\
\hline \multicolumn{9}{|c|}{$A B I$} \\
\hline Marzo & 28.8 & Moderada & 37 & Moderada & 99.4 & Muy buena & 125.8 & Muy buena \\
\hline Mayo & 50.8 & Buena & 81 & Muy buena & 98.6 & Muy buena & 115.4 & Muy buena \\
\hline Julio & 131 & Muy buena & 110.8 & Muy buena & 88.5 & Muy buena & 106.8 & Muy buena \\
\hline Setiembre & 103.4 & Muy buena & 141 & Muy buena & 130.4 & Muy buena & 139 & Muy buena \\
\hline Noviembre & 76.6 & Muy buena & 112.8 & Muy buena & 58.8 & Buena & 85 & Muy buena \\
\hline Enero & 70.6 & Buena & 77.6 & Muy buena & 80.6 & Muy buena & 109 & Muy buena \\
\hline
\end{tabular}

Valcárcel Rojas (2011) y en tributarios de Madre de Dios por Guevara Córdova (2013), señalaron una reducción del caudal en la temporada seca; concordando con lo observado en el área estudiada. Estos cambios hidrológicos definen las características físicas del medio acuático cambiando la disponibilidad de los diferentes microhábitats (DávilaRecinos et al., 2019; Allan \& Castillo, 2007). Los parámetros fisicoquímicos como el $\mathrm{pH}$, temperatura, salinidad, sólidos totales presentaron poca variación a lo largo del año de estudio a diferencia del oxígeno disuelto que sí disminuyó considerablemente en julio. Esta disminución en el oxígeno disuelto estaría asociado a la poca turbulencia debido a las escasas lluvias durante la temporada seca el cual influyó en el intercambio gaseoso del oxígeno entre el aire y agua (Mulholland et al., 2005).

La composición de la comunidad de macroinvertebrados acuáticos evidenció una alta riqueza comparado a lo registrado por Blanco (2005) y Salcedo \& Trama (2014) en Oxapampa. Estas diferencias podrían estar asociadas al esfuerzo de muestreo aplicado en este estudio, como la repetibilidad y el monitoreo en cuatro microhábitats diferentes a lo largo de un año. Asimismo, el microhábitat orgánico de hoja retenida en la corriente tuvo una alta abundancia ( $36 \%$ ) y riqueza de macroinvertebrados acuáticos (90 géneros) seguido por musgo (87 géneros), 
grava ( 82 géneros) y piedra ( 80 géneros). El microhábitat de hoja retenida en la corriente es uno de los microhábitats más abundantes en ecosistemas montanos y preferidos por los macroinvertebrados acuáticos, debido a que es una fuente importante de alimento y a su estabilidad y permanencia sobre todo en temporadas secas (Baptista et al., 2001; Wallace \& Webster, 1996; Huamantinco \& Nessimian, 1999).

En cuanto a los géneros más abundantes por microhábitat, se ha registrado en piedra a Gigantodax con la mayor abundancia relativa entre otros géneros como Baeodes y Farrodes (Ephemeroptera); y, Mortoniella, Helicopsyche y Leptonema (Trichoptera). La presencia de estos insectos en el microhábitat de piedra estaría relacionado a la forma de obtención de sus alimentos para lo cual algunos presentan adaptaciones como cepillos bucales (Gigantodax) para filtrar el agua además de presentar estructuras de fijación para sostenerse en un substrato duro como la piedra (Merritt, R. W.; K. W. Cummins \& Berg, 2008; Domínguez \& Fernández, 2009). En relación a los efemerópteros, su presencia estaría asociada a zonas con mucha oxigenación debido a que el sustrato pedregoso se ubica principalmente en la zona de rápidos dentro de las quebradas a la remoción constante del agua (Domínguez et al., 2006). Los Trichoptera registrados en este microhábitat estarían asociados a su forma de alimentación, ya que suelen raspar las superficies rocosas para la obtención de su alimento como los géneros de las familias Glossosomatidae (Mortoniella) y Helicopsychidae (Helicopsyche) (Tomanova et al., 2006).

En el microhábitat de grava el género Farrodes fue el más abundante aunque también destacaron Anchytarsus, Anacroneuria, Limnophila, Leptonema y Neoelmis. La presencia de estos géneros estaría relacionado a su alimentación, ya que este microhábitat concentra gran cantidad de material orgánico particulado grueso y fino producto del arrastre y deposición en estos espacios. Estos resultados concuerdan con Dávila-Recinos et al. (2019) quienes señalaron que algunos Ephemeroptera y Chironomidae aprovecharían el detritus acumulado en este microhábitat.

En el microhábitat de musgo se registró como el más abundante al género Rheotanytarsus (Chironomidae) seguido de Metrichia (Hydroptilidae). La preferencia de estos géneros por el microhábitat de musgo ya ha sido citada por diversos autores (Pinder, 1986; Merritt, R. W.; K. W. Cummins \& Berg, 2008; Maurer \& Brusven, 1983; Huamantinco \& Ortiz, 2011), quienes mencionan la importancia de estas briofitas para que estos insectos puedan construir sus estuches, además de permanecer fijados y no ser tan fácilmente desalojados por la corriente; donde el musgo adherido a las rocas y piedras en las quebradas, no se utiliza en sí mismo como alimento, sino como lugar donde se acumula alimento en la forma de detrito fino y además constituye un lugar de protección y refugio (Allan \& Castillo, 2007).

En cuanto a la hojarasca atrapada en la corriente los géneros Parametriocnemus (Chironomidae) y Phylloicus (Calamoceratidae) fueron los más resaltantes. La abundancia de este chironomidae estaría asociado a la alta concentración de detritus producto de la descomposición de hoja retenida en la corriente (Merritt et al. 2008) asociado a la gran disponibilidad de hojarasca a lo largo de la quebrada Pichita. La relación entre Phylloicus y la hojarasca atrapada en la corriente se debe a que estos insectos lo utilizan para su alimentación y construcción de sus estuches transportables (Oliveira \& Nessimian, 2010).

Los resultados encontrados en la quebrada Pichita evidencian que la mayoría de los macroinvertebrados acuáticos tienden a ocupar más de un tipo de microhábitat (Ramírez \& Gutiérrez-Fonseca, 2014) sin embargo se observan preferencias de estos organismos por algunos hábitat en particular tal es el caso, por ejemplo, de algunos géneros como Leptonema y Smicridea (familia Hydropsychidae) los que tendrían diferentes nichos ecológicos, siendo Leptonema muy abundante en los microhábitats de piedra, grava y hoja retenida en la corriente; y, Smicridea en el microhábitat de musgo. Respecto a lo registrado, Álvarez-Soraca et al. (2017) encontraron diferencias en las partes bucales de ambos géneros sugiriendo diferencias en la forma de obtención de sus alimentos.

Respecto a las métricas de bioindicación tanto el índice BMWP/Col y ABI registraron diferencias estadísticamente significativas según microhábitats estudiados, encontrándose marcadas diferencias entre los microhábitats de piedra-hoja y musgo-hoja. Estas diferencias están relacionadas a los organismos que se desarrollan en estos microhábitats y a sus valores de tolerancia evidenciando la importancia de los microhábitats inorgánicos y orgánicos en una evaluación. Por un lado el microhábitat de piedra, que a pesar de ser un substrato duro e inerte permite la fijación de algunas especies además de presentar espacios intersticiales alojando a la comunidad perifítica que sirve de alimento para los raspadores (Velásquez \& Miserendino, 2003), asimismo el microhábitat de grava también fue importante debido a que presenta material orgánico particulado grueso y fino traído por la corriente (Allan \& Castillo, 2007); y por otro lado los microhábitats orgánicos como el musgo y la hoja retenida en la corriente, que suelen dar estabilidad a muchas especies además de acumular partículas orgánicas en el caso del musgo o ser una fuente rica en nutrientes como la hoja. Estos resultados evidencian la importancia de considerar un monitoreo multihábitat tomando en cuenta todos los microhábitats presentes en un cuerpo de agua y obtener una riqueza representativa del área debido a la especificidad de algunas especies por determinados microhábitats, además la repetibilidad del muestreo podría asegurar la obtención de aquellas especies menos frecuentes, pero con puntuaciones importantes para el puntaje final de cada índice. La aplicación de los índices BMWP/Col y ABI parecen complementarse presentando puntuaciones y resultados de calidad acuática similares en la quebrada Pichita, lo cual podría considerarse como un precedente para gestionar y evaluar la calidad acuática en quebradas de bajo orden y ubicadas dentro de un ecosistema montano.

\section{CONCLUSIONES}

La presencia de diferentes microhábitats en la quebrada Pichita tuvo un efecto significativo sobre los valores de los índices $\mathrm{BMWP} / \mathrm{Col}$ y $\mathrm{ABI}$. El uso de ambos índices 
se complementa para determinar la calidad acuática en quebradas de primer orden de un ecosistema montano. La información generada en este estudio enfatiza la importancia de un muestreo multihábitat y repetitivo para una adecuada gestión del agua.

\section{AGRADECIMIENTOS}

La realización de este trabajo fue posible gracias al apoyo de la ONG APRODES por el permiso y acompañamiento durante las arduas tareas en campo.

\section{REFERENCIAS}

Acosta, R., Ríos, B., Rieradevall, M., \& Prat, N. (2009). Propuesta de un protocolo de evaluación de la calidad ecológica de ríos andinos (CERA) y su aplicación a dos cuencas en Ecuador y Perú. Limnetica, 28(1), 35-64. https://ddd.uab.cat/pub/ limnetica/02138409v28n1/02138409v28n1p35.pdf

Acosta Riveros, C. R. (2009). Estudio de la Cuenca Altoandina del Rio Canete (Peru): Distribucion altitudinal de la comunidad de macroinvertebrados bentonicos y characterizacion hidroquimica de sus cabeceras carsticas. Universitat de Barcelona, Tesis Doct, 1-153. https://dialnet.unirioja.es/ servlet $/$ tesis? $\operatorname{codigo}=254655$

Alba-Tercedor, J. (1996). Macroinertebrados acuaticos $y$ calidad de las aguas de los ríos. Instituto Tecnologico Geominero de Espana. https:// books.google.com.pe/books?hl=es \&lr=\&id=1blWQWRyloC\&oi=fnd\&pg=PA203\&dq=Alba-Tercedor,+19 96\&ots=VfQbH7Yuup\&sig=81POMKkRKPEYO2fDpio1A TURGig\# $\mathrm{v}=$ onepage $\& \mathrm{q}=$ Alba-Tercedor $\% 2 \mathrm{C} 1996 \& \mathrm{f}=$ false

Allan, J., \& Castillo, M. (2007). Stream ecology; Structure and Function of Running Waters. Springer 2nd Ed. Dordretc. http://41.89.141.8/kmfri/handle/123456789/1152

Álvarez-Soraca, K. D., Tamaris-Turizo, C. E., \& Guzmán-Soto, C. J. (2017). Morfología de las piezas bucales y hábitos alimenticios de Leptonema y Smicridea (Trichoptera: Hydropsychidae) del río Gaira, Sierra Nevada de Santa Marta, Colombia. Revista de Biologia Tropical, 65(4), 1231-1244. https://doi.org/10.15517/rbt.v65i4.26638

Baptista, D. F., Buss, D. F., Dorvillé, L. F., \& Nessimian, J. L. (2001). Diversity and habitat preference of aquatic insects along the longitudinal gradient of the Macaé River basin, Rio de Janeiro, Brazil. Brazilian Journal of Biology = Revista Brasleira de Biologia, 61(2), 249-258. https://doi org/10.1590/s0034-71082001000200007

Blanco, A. (2005). The impact of solid and liquid wastes from a rural town on the Chorobamba river, Oxapampa, peruvian amazon. https://digitalcommons.fiu.edu/etd/1692/

Brack Egg, A., \& Mendiola, C. (2000). Ecología del Perú. In Enciclopedia. https://www.peruecologico.com.pe/libro.htm

Dávila-Recinos, G., Ortiz, J. R., \& Reyes-Morales, F. (2019). Efecto del microhábitat sobre la abundancia y riqueza de macroinvertebrados bentónicos en dos ríos tropicales de montaña, Guatemala. Ciencia, Tecnología y Salud, 6(1), 7-21. https://digi.usac.edu.gt/ojsrevistas/index.php/cytes/ article/view/657
Domínguez, E., \& Fernández, H. R. (2009). Macroinvertebrados bentónicos Sudamericanos. Sistemática y Biología. Fundación Miguel Lillo Tucumán - Argentina. https:// www.researchgate.net/publication/260417584 Macroinvertebrados bentonicos Sudamericanos Sistematica_y_Biologia

Domínguez, E., Molineri, C., Pescador, M. L., Hubbard, M. D., \& Nieto, C. (2006). Ephemeroptera of South America. In Aquatic Biodiversity in Latin America Biodiversidad Acuatica en America Latina (Vol. 2, p. 646). Pensoft Publisher. https://books.google.com.pe/ books?hl=es\&lr $=\& \mathrm{id}=$ dwaJWle8OfkC\&oi $=$ fnd \&pg $=$ PA17 $\& \mathrm{dq}=$ Domínguez, + E.,+ Molineri, + C.,+ Pescador,+ M. + L., + Hubbard,+M.+D., $+\% 26+$ Nieto, + C. $+(2006) .+$ Ephemero ptera + of + South + America. + In + Aquatic + Biodiversity + in + Latin + America + Biodiversidad + Acuatica + en + America + Latina $+($ Vol. $+2,+$ p. +646$) .+\&$ ots $=$ BsJwKBESHG\&sig $=$ onUEkTpQH9yygOqOi2ovPvtmnqQ\#v=onepage\&q\&f $=$ false

Epler, J. H. (1995). Identification manual for the larval Chironomidae (Diptera) of Florida. In ... manual for the larval Chironomidae (Diptera) of Florida .... http://www. cabdirect.org/abstracts/19960503385.html

Graça, M. A.S., Ferreira, R. C. F., \& Coimbra, C. N. (2001). Litter processing along a stream gradient: The role of invertebrates and decomposers. Journal of the North American Benthological Society, 20(3), 408-420. https:// doi.org/10.2307/1468038

Graça, Manuel A.S. (2001). The role of invertebrates on leaf litter decomposition in streams - A review. In International Review of Hydrobiology (Vol. 86, Issues 4-5, pp. 383-393). https:// doi.org/10.1002/1522-2632(200107)86:4/5<383::AIDIROH383>3.0.CO;2-D

Guevara Córdova, C. H. (2013). Evaluación de la comunidad de macroinvertebrados de bancos vegetados en quebradas contaminadas por minería aurifera. Madre de Dios-Perú. https://cybertesis.unmsm.edu.pe/handle/20.500.12672/3431

Hamada, J. L., Nessimian, N., \& Querino, R. B. (2019). Insetos aquáticos na Amazônia brasileira: taxonomia, biologia e ecologia. Editora INPA. https://www.embrapa.br/buscade-publicacoes/-/publicacao/1000609/insetos-aquaticos-naamazonia-brasileira-taxonomia-biologia-e-ecologia

Holdridge, L. R. (2013). Ecología basada en zonas de vida. In 1982 (Vol. 53, Issue 9). https://repositorio.iica.int/ handle/11324/7936

Huamantinco, A. A., \& Nessimian, J. L. (1999). Estrutura e distribuição espacial da comunidade de larvas de Trichoptera (insecta) em um tributario de primeira ordem do rio Paquequer, Teresópolis, RJ. Acta Limnologica Brasiliensia, 11(2), 1. https://www.ablimno.org.br/acta/pdf/ acta_limnologica_contents1102E_files/resumo 1_11(2).pdf

Huamantinco, A., \& Ortiz, W. (2011). Clave de géneros de larvas de Trichoptera (Insecta) de la Vertiente Occidental de los Andes, Lima, Perú. Revista Peruana de Biología, 17(1). https://doi.org/10.15381/rpb.v17i1.54

Huaylinos Villalva, W., Quispitúpac Quispitúpac, E., \& Martínez Luján, N. (2003). Variabilidad fisicoquímica y fisiográfica del ecosistema de manglar San Pedro-Vice (Piura-Perú). Revista Del Instituto de Investigación de La Facultad de Ingeniería Geológica, Minera, Metalurgica y Geográfica, 
6(11), 7-19. https://revistasinvestigacion.unmsm.edu.pe/ index.php/iigeo/article/view/517

Lovera, D., Zavaleta, A., Puente, L., Quiñones, J., Gagliufi, P., Coronado, R., Arias, V., Terrel, M., Revilla, A., Diego, J., Semino, C. (2008). Hidrometalurgia de minerales sulfurados con presencia de bacterias en medio salinos. Revista Del Instituto de Investigación de La Facultad de Ingeniería Geológica, Minera, Metalurgica y Geográfica, 11(22), 7-12. https://revistasinvestigacion.unmsm.edu.pe/index. php/iigeo/article/view/382

Maestre, J. A., \& Carranza, C. C. (2018). Macroinvertebrados acuáticos y caracterización ecológica de los ambientes dulceacuícolas del área de influencia del gasoducto PERU LNG en los departamentos de Ica y Huancavelica. Revista Del Instituto de Investigación de La Facultad de Ingeniería Geológica, Minera, Metalurgica y Geográfica, 20(40), 8693. https://revistasinvestigacion.unmsm.edu.pe/index.php/ iigeo/article/view/14394

Manzo, V., \& Archangelsky, M. (2008). A key to the known larvae of South American Elmidae (Coleoptera: Byrrhoidea), with a description of the mature larva of Macrelmis saltensis Manzo. Annales de Limnologie, 44(1), 63-74. https://doi. org/10.1051/limn:2008023

Manzo, Verónica. (2005). Key to the South America genera of Elmidae (Insecta: Coleoptera) with distributional data. Studies on Neotropical Fauna and Environment, 40(3), 201-208. https://doi.org/10.1080/01650520500140619

Maurer, M. A., \& Brusven, M. A. (1983). Insect abundance and colonization rate in Fontinalis neo-mexicana (Bryophyta) in an Idaho Batholith stream, U.S.A. Hydrobiologia, 98(1), 9-15. https://doi.org/10.1007/BF00019246

Merritt, R. W.; K. W. Cummins \& Berg, M. B. (2008). An introduction to the aquatic insects of North America. Dubuque [U.S.A.] : Kendall Hunt, 1158. https://catalogo.latu.org.uy/ opac_css/index.php?lvl=notice_display\&id=2669

Meza-S, A. M., Rubio-M, J., G-Dias, L., \& M-Walteros, J. (2012). Calidad de agua y composición de macroinvertebrados acuáticos en la subcuenca alta del río Chinchiná. Caldasia, 34(2), 443-456. https://www.jstor.org/ stable/23641966?seq=1

Mulholland, P. J., Houser, J. N., \& Maloney, K. O. (2005). Stream diurnal dissolved oxygen profiles as indicators of in-stream metabolism and disturbance effects: Fort Benning as a case study. Ecological Indicators, 5(3), 243-252. https://doi. org/10.1016/j.ecolind.2005.03.004

Oliveira, A. L. H. de, \& Nessimian, J. L. (2010). Spatial distribution and functional feeding groups of aquatic insect communities in Serra da Bocaina streams, southeastern Brazil. Acta Limnologica Brasiliensia, 22(4), 424-441. https://doi.org/10.4322/actalb.2011.007

Ottoboni Segura, M., Valente-Neto, F., \& Fonseca-Gessner, A. A. (2011). Elmidae (Coleoptera, Byrrhoidea) larvae in the state of São Paulo, Brazil: Identification key, new records and distribution. ZooKeys, 151, 53-74. https://www.ncbi.nlm. nih.gov/pmc/articles/PMC3286224/

Passos, M. I. S., Nessimian, J. L., \& Ferreira Junior, N. (2007). Chaves para identificação dos gêneros de Elmidae (Coleoptera) ocorrentes no Estado do Rio de Janeiro, Brasil. Revista Brasileira de Entomologia, 51(1), 42-53. https:// doi.org/10.1590/s0085-56262007000100008
Pinder, L. C. V. (1986). Biology of freshwater Chironomidae. Annual Review of Entomology. Vol. 31, 1-23. https://doi. org/10.1146/annurev.en.31.010186.000245

Ramírez, A., \& Gutiérrez-Fonseca, P. E. (2014). Functional feeding groups of aquatic insect families in Latin America: A critical analysis and review of existing literature. Revista de Biologia Tropical, 62, 155-167. https://doi.org/10.15517/ rbt.v62i0.15785

Ramírez, A., Paaby, P., Pringle, C. M., \& Agüero, G. (1998). Effect of habitat type on benthic macroinvertebrates in two lowland tropical streams, Costa Rica. Revista de Biologia Tropical, 46(SUPPL. 6), 201-213. https://doi.org/10.15517/ rbt.v46i6.29826

Reynel, C. (2012). Flora y fauna del bosque montano nublado puyu sacha. Asociación Peruana Para La Promoción Del Desarrollo Sostenible APRODES, 54(1), 383. http://www. aprodes.org/pdf/flora fauna.pdf

Roldán-Pérez, G. (2016). Los macroinvertebrados como bioindicadores de la calidad del agua: cuatro décadas de desarrollo en Colombia y Latinoamerica. Rev. Aca. Colomb. Ciene. Ex. Fis. Nat., 40(155), 254-274. https://doi. org/10.18257/raccefyn.335

Roldán, G. (2003). Bioindicación de la calidad del agua en Colombia: propuesta para el uso del método BMWP Col. In Bioindicación de la calidad del agua en Colombia: propuesta para el uso del método BMWP Col (p. 170). https://books.google.com.pe/books? $\mathrm{hl}=\mathrm{es} \& \mathrm{lr}=\& \mathrm{id}=\mathrm{ZEjgIKZTF} 2 \mathrm{UC} \& \mathrm{oi}=\mathrm{fnd} \& \mathrm{pg}=\mathrm{PA} 1 \&$ dq=Roldán, + G. $+(2003) .+$ Bioindicación + de + la + calidad + del+agua + en + Colombia:+propuesta + para + el + uso + del + método+BMWP/Col.+Colección + Ciencia $+y+$ Tecnología, + Editorial+Universidad+de+Antioquia

Ruiz-Moreno, J., Ospina-Torres, R., Gómez-Sierra, H., \& Riss, W. (2000). Guía para la identificación genérica de larvas de quironómidos (Diptera: Chironomidae) de la Sabana de Bogotá. III subfamilias Tanypodinae, Podonominae y Diamesinae. Caldasia, 22(1), 34-60. https://revistas.unal. edu.co/index.php/cal/article/view/17550/18401

Salcedo, S. A., \& Trama, F. A. (2014). Manual de Identificación de Macroinvertebrados Acuáticos de la Microcuenca San Alberto, Provincia de Oxapampa, Perú (CONCYTEC (ed.)). https://isbn.cloud/9789972501715/manual-deidentificacion-de-macroinvertebrados-acuaticos-de-lamicrocuenca-san-alberto-provincia/

Thorp, J.H., \& A. P. C. (2018). Thorp and Covich's Freshwater Invertebrates. In Thorp and Covich's Freshwater Invertebrates. https://doi.org/10.1016/c2015-0-01626-4

Tomanova, S., Goitia, E., \& Helešic, J. (2006). Trophic levels and functional feeding groups of macroinvertebrates in neotropical streams. Hydrobiologia, 556(1), 251-264. https://doi.org/10.1007/s10750-005-1255-5

Valcárcel Rojas, D. R. (2011). Evaluación de la degradación de ecosistemas dulceacuícolas en la cuenca baja del río Uctubamba (Amazonas - Perú) mediante el uso de macroinvertebrados bentónicos. Tesis. https://cybertesis. unmsm.edu.pe/handle/20.500.12672/1356

Velásquez, S. M., \& Miserendino, M. L. (2003). Análisis de la materia orgánica alóctona y organización funcional de macroinvertebrados en relación con el tipo de hábitat en ríos 
de montaña de Patagonia. Ecologia Austral, 13(1), 67-82. https://www.researchgate.net/publication/262590269 Analisis de la materia organica aloctona y organizacion funcional_de_macroinvertebrados_en_relacion_con_el_ tipo_de_habitat_en_rios_de_montana_de_Patagonia
Wallace, J. B., \& Webster, J. R. (1996). The role of macroinvertebrates in stream ecosystem function. Annual Review of Entomology, 41(1), 115-139. https://doi. org/10.1146/annurev.en.41.010196.000555 
